\title{
Fox family ties
}

Cell Research (2013) 23:452-454. doi:10.1038/cr.2013.5; published online 15 January 2013

The transcription factor Foxp3 plays an indispensible role in the differentiation of regulatory $T$ (Treg) cells and the expression of their suppressive functions. In a recent article published in Nature, Ouyang et al. demonstrate that Treg cell differentiation is also enabled by transcriptional networks controlled by another Forkhead box family member, Foxo1.

From flies to mammals, the forkhead box (Fox) family of transcription factors promotes the integration of environmental cues with cell metabolism and homeostasis [1]. In humans and mice, inactivation of the gene encoding the Foxp subfamily member 3 (Foxp3) results in aggressive, fatal multi-organ autoimmunity [2]. Following these observations, Foxp3 was quickly shown to be essential for the development and function of regulatory $\mathrm{T}$ (Treg) cells. However, experiments that replaced Foxp3 with EGFP or a non-functional Foxp3-EGFP fusion protein showed that developing Treg cells, although lacking regulatory function, retained a portion of the Treg cell transcriptome [3, 4]. These observations suggested that a higher order of transcriptional regulation was necessary in addition to Foxp3 expression for Treg cell development.

Additional Fox subfamily members (Foxo1 and Foxo3) are also expressed in lymphocytes, including $\mathrm{T}$ cells. Ablation of Foxo3 has little effect on $\mathrm{T}$ cell function and may result in a mild lymphproliferative and inflammatory syndrome, depending on the investigational approach $[5,6]$. In contrast, $\mathrm{T}$ cell-specific deletion of Foxol produces peripheral $\mathrm{T}$ cell activation, increases the number of $\mathrm{T}$ cells producing IFN- $\gamma$ and IL-17A, and increases autoantibody production [7, 8]. T cells in Foxo1-deficient mice have low levels of IL-7R $\alpha$ and CD62L, which reduces naïve $T$ cell survival. However, there is little effect of $\mathrm{T}$ cell-specific Foxo1 deficiency on thymic development, and the frequency of Foxp $3^{+}$cells in the thymus, spleen, and lymph nodes of these mice is similar to controls. Importantly, mice with a compound deletion of Foxo 1 and Foxo3 in T cells develop a lethal autoimmune inflammatory disease associated with reductions in Treg cell frequency, number, and function [9]. The cumulative data support the hypothesis that Foxo1 and Foxo3 act early in Treg cell lineage specification, while Foxp3 is a late-acting differentiation factor that maintains and stabilizes the Treg cell phenotype. Although expression of Foxol is upregulated in mature thymocytes and Foxol is expressed in peripheral Treg cells, the role of Foxo1 beyond Treg cell lineage specification has not been explored.

A recent paper by Ouyang et al. [10] has dissected the impact of Foxo1 expression on Treg cell function. In quiescent $\mathrm{T}$ cells, Foxol is found in the nucleus and shuttled to the cytoplasm following TCR engagement [1]. To interrogate the kinetics of Foxo1 translocation, the authors engineered a reporter allele that expresses Foxo1 fused in-frame to a green fluorescent protein, Flag, and a biotin-labeling peptide $\left(\right.$ Foxo $\left.^{\text {tag }}\right)$. A Foxp $3^{\text {RFP }}$ reporter allele was used in combination to mark Treg cells. When TCR stimulation was provided by high-dose anti-CD3 antibody, clearance of Foxol from the nucleus of all conventional $\mathrm{T}$ (Tconv) and Treg cells was observed. In contrast, low-dose anti-CD3-mediated stimulation triggered translocation of Foxo1 in Tconv cells but not in Treg cells. Attenuated Foxo1 and Akt phosphorylation was also seen in low-dose anti-CD3stimulated Treg cells, consistent with regulation of Foxo1 nuclear export by Akt-induced Foxo1 phosphorylation. Thus, under conditions of low-dose anti-CD3 TCR crosslinking, Treg cells demonstrated attenuated Akt activation and Foxo1 nuclear clearance.

Next, Ouyang et al. observed that conditional ablation of Foxo1 in Treg cells (Foxp $3^{\text {cre }}$ Foxo $^{\text {ftfl }}$ mice) resulted in lymphoproliferation, leukocyte infiltration of multiple tissues, and a rapidly fatal phenotype reminiscent of mice with Foxp3 deficiency. The majority of $\mathrm{CD}^{+}{ }^{+}$and $\mathrm{CD} 8^{+} \mathrm{T}$ cells were $\mathrm{CD} 62 \mathrm{~L}^{\text {lo }}$ CD44 ${ }^{\text {hi }}$ and produced IFN- $\gamma$. In 12-dayold Foxp $3^{\text {cre }}$ Foxo $1^{f l f l}$ mice, Treg cell numbers were normal in the thymus and spleen but increased in peripheral tissues by 20 days, consistent with a loss of Treg cell function. Taken together, the preceding observations suggest that Foxo1-dependent regulation of gene expression is essential for Treg cell suppressive function.

Cytosolic Fox family proteins are not inert and may interact with other transcription factors or may regulate transcription-independent cellular processes [1]. Accordingly, the nuclear and cytosolic activities of Foxo1 were dissected with a mutant Foxol allele engineered to eliminate Akt phosphorylation sites (FOXO1 $\left.{ }^{A A A}\right)$ and thereby block nuclear export. This mutant allele containing a loxp-flanked 'STOP' cassette was inserted into the Rosa26 
locus. Expression of the Foxo $1^{A A A}$ allele in Foxp $3^{\text {cre }} \mathrm{FoxO}^{\mathrm{Alfl}}$ mice rescued the lethal inflammatory phenotype, linking the nuclear activity of Foxo1 with Treg cell function. Indeed, chromatin immunoprecipitation experiments identified more than 3000 putative Foxol binding sites, enriched in promoters and $5^{\prime}$ untranslated regions. These included the known sites in the Foxp3 locus and $I L-7 \mathrm{ra}$ enhancer (Figure 1). Transcriptional profiling of Treg cells from wild-type, Foxp $3^{\text {cre }}$ Foxol $^{\text {flf }}$, Fox$\mathrm{p}^{\text {cre }} \mathrm{FoxO}^{\text {AAA/+ }}$, and Foxp $3^{\text {cre }} \mathrm{Foxo}^{\mathrm{Al}}$ ${ }^{A}$ Foxo $1^{A A A /+}$ mice cross-referenced with the Foxo1 binding site data identified 310 direct target genes, many of which were associated with Jak-STAT, TCR and insulin signaling pathways. Interestingly, just $7 \%-10 \%$ of Foxo1-bound genes were also occupied by Foxp 3 in other studies [11]. One of these genes, Ctla4, contributes to Treg cell suppressive function and is reported to be a direct target of both Foxp3 and Foxo1 [12]. In the current study, however, Treg cell-specific Foxo1 deficiency had minimal effects on CTLA4 expression and did not affect Treg cell-mediated in vitro suppression. Overall, the authors conclude that the majority of the Foxp3dependent transcriptional program is intact in Foxo1-deficent mice, and that the loss of suppressive activity is not readily attributed to reductions in CTLA4.

Ouyang et al. also find that Foxo1 is recruited to a regulatory element near the Ifng transcriptional start site. In a fraction of Foxo1-deficient but not WT Treg cells, IFN- $\gamma$ was induced after stimulation with IL-2, IL-12, and IFN- $\gamma$. This was not the case with WT and Foxo1-deficient Tconv cells, which produced similar amounts of IFN- $\gamma$ under the same stimulating conditions. These data are consistent with a previous report showing that TGF- $\beta$ signaling fails to repress the transcription factor T-bet in Foxo1-deficient $\mathrm{T}$ cells. To determine whether the IFN- $\gamma$ produced by Foxo1-deficient Treg cells was related to their decreased

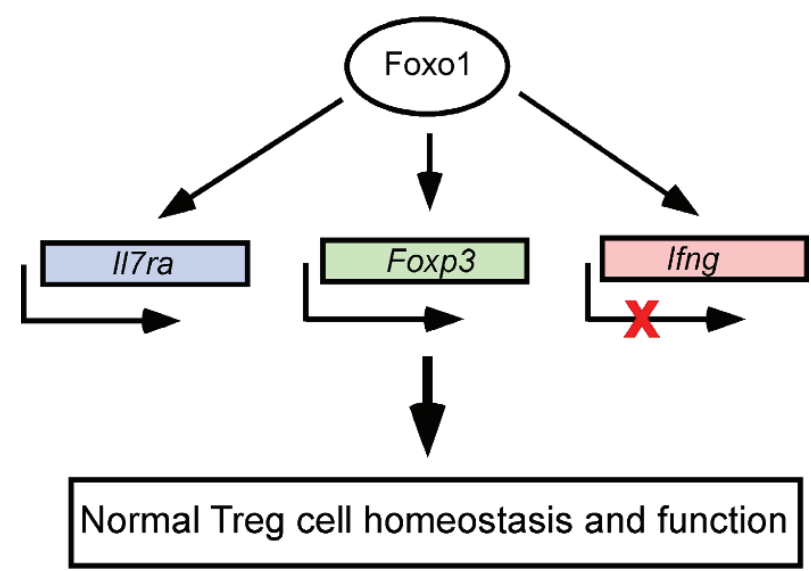

Figure 1 Foxo1 target genes support Treg cell development and function. Foxo1 binds to the II7ra enhancer and controls IL-7R $\alpha$ chain expression. Other Foxo1 target genes include Foxp3 and Ifng. Foxo1 regulation of Foxp3 may be relevant in development of Treg cells in the thymus, and appears to also regulate TGF- $\beta$-mediated induction of Foxp3 in mature peripheral T cells. Foxo1 repression of Ifng expression is required for normal Treg cell suppressive function.

suppressive function, the authors used a lymphopenia-associated transfer model of colitis. Transfer of $\mathrm{FoxO}^{-/<}$or Foxo $1^{--}$Ifng $^{--}$Treg cells into Rag $1^{--}$ mice did not result in colitis, suggesting that the Foxo $1^{-/-}$Ifng $^{--}$Treg cells have no autonomous pathogenic potential in this assay. However, only the Foxol $1^{-/}$ Ifng $^{-/}$but not the Foxol ${ }^{-/}$Treg cells prevented colitis when co-transferred with naïve $\mathrm{T}$ cells. Thus, the absence of IFN- $\gamma$ in $\mathrm{FoxO}^{-/-}$Treg cells partially restored their suppressive function, suggesting that Foxo1-mediated inhibition of IFN- $\gamma$ expression is required for Treg cell function.

The emerging view from the body of work examining the role of the Foxo family of transcription factors points to an essential role for Foxo1 in Treg cell biology both during development in the thymus and upon egress to the periphery. Foxo 1 appears to enable TGF- $\beta$ signaling in Treg cells and prevent the degeneration of the Treg cells into a pro-inflammatory Th1 phenotype with high IFN- $\gamma$ production. The finding that the majority of Foxo1-regulated genes in Treg cells did not overlap those regulated by Foxp3 points to mostly distinct transcriptional networks oper- ated by the respective factors to ensure the fidelity of the Treg cell phenotype. Several questions regarding the role of Foxo proteins in the development and functions of Treg cells remain unanswered. The precise mechanism(s) by which Foxo proteins contribute to Treg cell development remain undefined. Also, IFN- $\gamma$ deficiency ameliorated but did not fully rescue the disease of mice with Foxo1-deficient Treg cells, indicating that other mechanisms exist. Finally, defects in the Foxo pathway may underlie hitherto undiscovered Mendelian cases of lymphoproliferation and autoimmunity. Future studies on the subject are bound to yield important insights into the role of this pathway in peripheral tolerance.

\section{Calvin B Williams ${ }^{1}$, Talal A Chatila ${ }^{2}$}

${ }^{I}$ Section of Rheumatology, Department of Pediatrics, Medical College of Wisconsin, Milwaukee, WI 53226, USA; '2Division of Immunology, the Children's Hospital, and the Department of Pediatrics, Harvard Medical School, Boston, MA 02115, USA

Correspondence: Calvin B Williams

E-mail: cwilliam@mcw.edu 


\section{References}

1 Ouyang W, Li MO. Trends Immunol 2010; 32:26-33.

2 Rudensky AY. Immunol Rev 2011; 241:260268.

3 Gavin MA, Rasmussen JP, Fontenot JD, et al. Nature 2007; 445:771-775.

4 Lin W, Haribhai D, Relland LM, et al. Nat
Immunol 2007; 8:359-368.

5 Lin L, Hron JD, Peng SL. Immunity 2004; 21:203-213.

6 Dejean AS, Beisner DR, Ch'en IL, et al. Nat Immunol 2009; 10:504-513.

7 Ouyang W, Beckett O, Flavell RA, et al. Immunity 2009; 30:358-371.

8 Kerdiles YM, Beisner DR, Tinoco R, et al. Nat Immunol 2009; 10:176-184.
9 Ouyang W, Beckett O, Ma Q, et al. Nat Immunol 2010; 11:618-627.

10 Ouyang W, Liao W, Luo CT, et al. Nature 2012; 491:554-559.

11 Zheng Y, Josefowicz SZ, Kas A, et al. Nature 2007; 445:936-940.

12 Kerdiles YM, Stone EL, Beisner DR, et al. Immunity 2010; 33:890-904. 